\title{
SUSTITUCIÓN DE LARDO POR GRASA VEGETAL EN SALCHICHAS: INCORPORACIÓN DE PASTA DE AGUACATE. EFECTO DE LA INHIBICIÓN DEL OSCURECIMIENTO ENZIMÁTICO SOBRE EL COLOR ${ }^{1}$
}

\author{
Ubaldo RUEDA-LUGO², Roberto GONZÁLEZ-TENORIO , Alfonso TOTOSAUS²,*
}

\begin{abstract}
RESUMEN
La sustitución de grasa animal es importante para mejorar la calidad nutricional de los alimentos de origen animal. Tradicionalmente, los embutidos contienen cantidades relativamente altas de grasas insaturadas, por lo que se ha buscado la sustitución parcial o total de estas con grasas o aceites de origen vegetal. El aprovechamiento del aguacate como fuente de grasa vegetal es una alternativa para este tipo de productos. La oxidación de este fruto es uno de los principales problemas durante la industrialización. En este trabajo se han añadido dos inhibidores del oscurecimiento enzimático, ácido ascórbico y eritorbato de sodio, para determinar el efecto de éstos sobre el color de salchichas de cerdo. Se han encontrado diferencias en la luminosidad de las muestras con respecto al tratamiento control, además de que este mismo parámetro se ha reducido con el tiempo de almacenamiento. No ha habido efecto significativo de los antioxidantes sobre las otras componentes del color. El uso de eritorbato de sodio en la elaboración de pasta de aguacate ha reducido el oscurecimiento de la pasta durante su congelación, sin afectar mayormente otros parámetros de color del producto terminado. La aceptación del producto por un grupo de consumidores ha sido aceptable.

Palabras-claves: sustitución de grasa, salchichas, aguacate, color.
\end{abstract}

\section{RESUMO}

SUBSTITUIÇÃO DO TOUCINHO POR GORDURA VEGETAL EM SALSICHAS: ADIÇÃO DA PASTA DE ABACATE. EFEITO DA INIBIÇÃO DO ESCURECIMIENTO ENZIMATICO NA COR. A substituição da gordura animal ou toucinho é importante para melhorar a qualidade nutricional dos alimentos de origem animal. Tradicionalmente, os embutidos contêm quantidades relativamente elevadas de gorduras insaturadas, e a substituição parcial ou total com gordura vegetal ou óleo é desejável. O abacate é fonte de gordura vegetal como uma alternativa para este tipo de produto. A oxidação desta fruta é um dos problemas principais durante a industrialização. Neste trabalho foram adicionados dois inibidores de escurecimento enzimatico, ácido ascórbico e eritorbato do sódio, para determinar o efeito destes na cor das salsichas. As diferenças na luminosidade das amostras foram detectadas em relação ao controle, além da diminução deste mesmo parâmetro com o tempo de armazenamento. Nenhum efeito significativo foi detectado nos outros parâmetros da cor do produto terminado. O uso do eritorbato do sódio na elaboração da pasta do abacate reduziu seu escurecimento durante o armazenamento, sem nenhum efeito nos outros parâmetros da cor. A aceitação do produto por um grupo de consumidores foi favorável.

Palavras-chave: substituição da gordura, salsichas, abacate, cor.

\section{INTRODUCCIÓN}

La mayoría de los productos cárnicos procesados contienen en su formulación concentraciones relativamente altas de grasas insaturadas, por lo que muchas veces su consumo se ve restringido por cuestiones de salud. Una alternativa para reducir o mejorar el balance de ácidos grasos es la incorporación de grasas o aceites de origen vegetal.

La sustitución de la grasa animal por aceites vegetales en productos cárnicos ha sido estudiada. En algunos países europeos, el aceite de soya ha sido utilizado como sustituto de la grasa en embutidos. MUGUERZA et al. [1] reemplazaron grasa dorsal de cerdo con aceite de oliva en salchichas secas fermentadas de un 10 al 20\%. Después de 4 semanas de fermentación los niveles de grasa afectaron la pérdida de peso y luminosidad de las muestras. El reemplazar el

\footnotetext{
${ }^{1}$ Recebido para publicação em 10/10/2005 Aceito para publicação em 3/5/2006 (001618)

${ }^{2}$ Laboratorio de Alimentos, Tecnológico de Estudios Superiores de Ecatepec. Avenida Tecnológico y Av. Central s/n, Ecatepec 55210, Estado de México, México.E-mail: totosaus@att.net.mx

${ }^{2}$ Instituto de Ciencias Agropecuarias, Universidad Autónoma del Estado de Hidalgo. Avenida Universidad km 1, Tulancingo 43000, Hidalgo, México. *A quem a correspondência deve ser enviada.
}

$20 \%$ de la grasa dorsal de cerdo afectó significativamente la luminosidad y amarillosidad de las salchichas. También se ha sustituido la grasa de cerdo por aceite de oliva en productos tipo salami. La sustitución parcial de la grasa de cerdo no ha afectado las características químicas, físicas y sensoriales de los productos, excepto la actividad de agua y la textura, pero sin afectar su vida de anaquel [2]. KAYAARDI \& GÖK [3] sustituyeron hasta un $60 \%$ de la grasa de res en un embutido tradicional turco "soudjouk", con aceite de oliva como grasa preemulsionada con aislado de proteína de soya. El colesterol disminuyó conforme la cantidad de aceite de oliva incorporada, lo que elevó asimismo los niveles de ácido oleico y linoleico. Al reemplazar $60 \%$ de la grasa de res no se encontró efecto sobre la textura, y el $40 \%$ de sustitución no alteró la aceptación general ni el color de las muestras. El uso de otros aceites, como el aceite de semilla de soya, aceite de girasol, de semilla de algodón, de maíz dulce o palmina se utilizaron para sustituir grasa en salchichas de res y salamis cocidos. La estabilidad de la emulsión de los productos fue buena. Sin embargo, la firmeza, luminosidad y color interno de las muestras se vio reducido [4]. El uso de aceites de palma en la sustitución de grasa de salchichas de pollo no afectaron los rendimientos de las muestras. La incorporación de diferentes 
tipos y niveles de aceite de palma resultaron en cambios significativos en el color (luminosidad, rojo y amarillo) de las salchichas [5]. PARK, RHEE \& ZIPRIN [6] substituyeron el lardo en salchichas bajas en grasa con aceite de girasol alto en ácido oleico, sin encontrar diferencias en el rendimiento entre estas formulaciones y el control, además de presentar propiedades de textura similares.

La incorporación de pasta de aguacate en salchichas de cerdo emulsionadas es una alternativa saludable para enriquecer el valor nutricional de este tipo de productos. $\mathrm{El}$ aguacate es el fruto del aguacatero (Persea americana), originario de México y Guatemala. La característica alimentaria principal de este fruto es su riqueza en grasas que van del 10-30 \%. La ventaja de estas grasas es que están formadas por ácidos grasos como los ácidos oleico, linoleico y palmítico, principalmente, por lo que puede ayudar a contrarrestar los efectos perniciosos de las grasas saturadas contenidas en las grasas animales [7]. Sin embargo, el aguacate tiende a sufrir reacciones de oscurecimiento al ser expuesto al aire. El oscurecimiento enzimático ocurre en algunas frutas y vegetales cuando el tejido es lastimado, cortado, pelado o expuesto a condiciones anormales. El tejido dañado rápidamente se obscurece debido a la conversión de los compuestos fenólicos a melaninas color café [8]. La actividad de la fenolasa resulta indeseable durante el procesamiento de frutas y verduras, por lo que su inhibición es importante, mediante la adición de acidulantes, como el ácido cítrico [9]. Otro compuesto utilizado es el ácido eritórbico o eritorbato de sodio, que realiza las mismas funciones del ácido ascórbico pero no tiene valor como vitamina [10].

El objetivo de este trabajo fue el de comparar dos aditivos inhibidores del oscurecimiento enzimático, ácido cítrico y eritorbato de sodio, durante la elaboración y almacenamiento congelado de pasta de aguacate, para ser utilizadas como sustituto de grasa animal en salchichas de cerdo, determinando su efecto sobre el color de las muestras finales.

\section{MATERIALES Y MÉTODOS}

\section{1- Elaboración de la pasta de aguacate}

Para evitar la oxidación u oscurecimiento enzimático en la pasta de aguacate durante su elaboración se utilizaron tres diferentes tratamientos incorporando $0.1 \%(\mathrm{p} / \mathrm{p})$ de ácido cítrico (J.T. Baker, Xalostoc, México) o eritorbato de sodio (Nutri-Fam, S.A. de C.V., Ciudad de México), respectivamente, como antioxidantes, y un tratamiento como control sin ningún antioxidante añadido. Las pastas fueron elaboradas a partir de aguacates frescos y maduros, retirándoles la cáscaras el hueso, repartiéndolas al azar entre los tratamientos para homogenizar con el respectivo antioxidante. Las pastas fueron congeladas inmediatamente después de elaboradas a $-20{ }^{\circ} \mathrm{C}$ en bolsas de polietileno y fueron utilizadas después de 3 días y antes de una semana en la formulación de las salchichas.

\section{2 - Fabricación de las salchichas}

Las salchichas fueron elaboradas de acuerdo a la formulación descrita en la Tabla 1. Cabe mencionar que la coloración final de este producto es de un verde claro debido al aguacate y al cilantro utilizado. La carne y la grasa dorsal (lardo) de cerdo fueron obtenidas de carnicerías locales y mantenidas en refrigeración hasta su uso. Se integró la carne con los ingredientes de la formulación más la mitad del hielo en un procesador de alimentos Moulinex ATX-41 durante 2-3 minutos. Se adicionó la pasta de aguacate de los diferentes tratamientos y el lardo en una proporción de 1:1.3 (p/p), determinada por investigaciones previas, con el resto de hielo y se homogenizó por 5 minutos controlando la temperatura del batido a $10 \pm 2{ }^{\circ} \mathrm{C}$. Los batidos fueron embutidos en tripas de celulosa y cocidos a $70{ }^{\circ} \mathrm{C}$ durante 15 min., enfriados en baño de hielo y almacenados a $4{ }^{\circ} \mathrm{C}$ en bolsas de polietileno para evitar pérdidas de humedad, muestreando a los 1, 3, 6 y 9 días. Se elaboraron dos lotes de $0.5 \mathrm{~kg}$ de cada tratamiento.

TABLA 1. Formulación de las salchichas con pasta de aguacate

\begin{tabular}{lc}
\hline Ingrediente & Porcentaje \\
\hline Carne de cerdo & 43.00 \\
Lardo & 13.00 \\
Pasta de aguacate & 17.00 \\
Hielo & 15.00 \\
K-Carragenina & 0.50 \\
Dextrosa & 0.25 \\
Fosfatos de sodio & 0.25 \\
Concentrado de suero de leche & 8.50 \\
Pimienta blanca & 0.10 \\
Cilantro & 0.15 \\
Sal cura & 0.50 \\
Sal & 2.25 \\
\hline
\end{tabular}

\section{3 - Determinación del color}

La luminosidad $\left(\mathrm{L}^{*}\right)$, componente verde (-a*) y componente amarilla ( $\left.b^{*}\right)$ del color en coordenadas CIE-Lab fueron determinadas en las pastas de aguacate y en la superficie de las salchichas de acuerdo a la metodología reportada por PAPADAKIS et al. [11] y YAM \& PAPADAKIS. [12], con algunas modificaciones. Las muestras fueron colocadas sobre cajas petri para capturar la imagen en un escáner plano Microtek Scanmaker 3600 (Microtek International, Inc.). El formato de color de la imagen fue cambiado en la pestaña de 'Image' del modo 'RGB' al modo 'Lab Color' en el software Adobe Photoshop v. 7.0 (Adobe System Inc.), de modo que se obtuvieron los parámetros de luminosidad ( $\left.\mathrm{L}^{*}\right)$, a (-a*, componente verde) y b ( $\mathrm{b}^{*}$, componente amarilla) del Histograma de la pestaña 'Image'. Los resultados son el promedio de tres lecturas de cada parámetro. Los valores se estandarizaron de acuerdo a las siguientes fórmulas (Equacións 1, 2 e 3): 


$$
\begin{gathered}
\mathrm{L}^{*}=\frac{\text { Luminosidad }}{255} \times 100 \\
\mathrm{a}^{*}=\frac{240 \mathrm{a}}{255}-120 \\
\mathrm{~b}^{*}=\frac{240 \mathrm{~b}}{255}-120
\end{gathered}
$$

\section{4 - Prueba de aceptación}

Debido a las características de este tipo de salchichas, es decir, su coloración verde por la incorporación de la pasta de aguacate y cilantro, se llevó a cabo una prueba de aceptación de las salchichas formuladas con eritorbato de sodio, puesto que este aditivo es un ingrediente utilizado en productos cárnicos curados para acelerar el proceso de curado, lo que mejora el rendimiento de oxido nítrico y reduce la posibilidad de formación de nitrosaminas [13]. El diseño del cuestionario se dividió en dos partes: la primera para conocer las expectativas del consumidor hacia este producto en particular; y la segunda para determinar el grado de aceptación de la salchicha. Se reclutó un total de 45 personas, como panel no entrenado, entre alumnos y personal del Tecnológico, con edades entre los 23 y 40 años, como potenciales consumidores con gusto por el consumo de productos cárnicos. Previamente a la prueba, los atributos a evaluar fueron descritos a los consumidores [14]: color, característico de acuerdo al producto; sabor, gusto salado característico percibido en el paladar; dureza, resistencia de la muestra a la fuerza ejercida para ser cortada o masticada; jugosidad, humedad residual de la muestra; y, aceptación general, la apreciación global de los atributos evaluados. Las muestras (2-3 g) se sirvieron a temperatura ambiente y se le solicitó al panelista que evaluara cada atributo marcando sobre una línea (escala no estructurada) de $10 \mathrm{~cm}$ de largo su apreciación de acuerdo a la escala "me agrada mucho" (extremo izquierdo) o "me desagrada mucho" (extremo derecho) [15].

\section{5 - Análisis estadístico}

El efecto de los tratamientos, tipo de antioxidante en la pasta y tiempo de almacenamiento sobre el color de las salchichas fue determinado mediante un análisis de varianza en el programa estadístico SAS v. 8.0 (SAS Institute, Cary, North Carolina), donde el modelo propuesto fue (Equación 4):

$y_{i j}=\mu+\alpha_{i}+\beta_{j}+\alpha \beta_{i j}+\epsilon_{i j}$

Donde $y_{i j}$ es el parámetro de color correspondiente para el $i$-ésimo tipo de antioxidante del $j$-ésimo día de almacenamiento; $\mu$ es la media total; $\alpha_{i}, \beta_{j}$ y $\alpha \beta_{i j}$ son los efectos principales por tipo de antioxidante, tiempo de almacenamiento y la interacción de primer orden entre los dos tratamientos; $\mathrm{y} \in_{i j}$ representa el error residual [16]. Diferencias significativas entre los tratamientos fueron determinadas mediante un análisis de medias de Duncan en el mismo programa.

\section{RESULTADOS Y DISCUSIÓN}

Dado que el aguacate es un producto perecedero y se necesita almacenar congelado para integrarse de esa manera a la formulación de las salchichas, la aplicación de antioxidantes durante su proceso se hace necesaria. Aunque sólo el exterior de las muestras almacenadas presentaba a simple vista cambios de coloración, el hecho de retirar esta parte de las muestras representa un desperdicio considerable, por lo que las muestras fueron totalmente homogeneizadas para las lecturas de color y para ser incorporadas en las formulaciones. Los valores de luminosidad de las pastas de aguacate fueron meno-

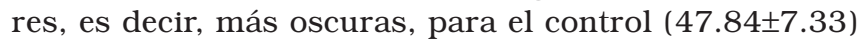
a la semana de almacenamiento en comparación con la pasta tratada con ácido ascórbico o eritorbato de sodio

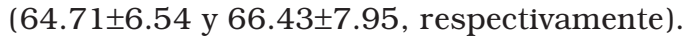

En cuanto al color superficial de las salchichas, se encontró un efecto significativo $(P<0.05)$ por el tipo de antioxidantes en la pasta de aguacate utilizada sobre la luminosidad de las salchichas, donde las muestras control fueron más luminosas que las muestras elaboradas a partir de pasta con los antioxidantes, porque la adición de ácido cítrico o eritorbato de sodio oscureció el color de las salchichas. Esto puede deberse a ligeros cambios en el $\mathrm{pH}$ por el uso del ácido cítrico o bien por la interacción del eritorbato de sodio con los nitratos durante la elaboración de las salchichas, con lo cual se forma una coloración más rosada, donde la coloración tuvo una tendencia más hacia el lado positivo de a (tonalidad roja), es decir, un valor menor de $-\mathrm{a}^{*}$. No hubo efecto significativo $(P>0.05)$ de los tratamientos sobre los parámetros de color verde y amarillo (Tabla 2). Se detectó además un efecto altamente significativo $(P<0.01)$ del tiempo de almacenamiento sobre la luminosidad de las muestras, donde con el paso del tiempo las salchichas se quedaron menos oscuras (valores mayores de $\mathrm{L}^{*}$ ), sobre todo las muestras control a partir de la semana seis, en los tres tratamientos. La componente verde del color $\left(-\mathrm{a}^{*}\right)$ se vio afectada significativamente $(P<0.05)$ por el tiempo de almacenamiento, tendiendo a aumentar progresivamente. No se detectó efecto significativo $(P>0.05)$ en la amarillosidad de las muestras (Tabla 2).

Los ácidos orgánicos han sido utilizados como acidulantes y conservadores. De manera particular, el ácido cítrico se utiliza como agente quelante de iones metálicos (particularmente cobre o hierro), que son poderosos agentes prooxidantes [17], y tienen también un efecto sinérgico con otras componentes antioxidantes [18]. Por otro lado, el eritorbato de sodio previene la oxidación de lípidos y pérdida de color en productos curados, puesto que su efecto protector está asociado a la prevención de 
TABLA 2. Análisis de medias de Duncan para los resultados de color de los diferentes tratamientos

\begin{tabular}{|c|c|c|c|c|}
\hline \multirow{2}{*}{ Tratamiento } & \multicolumn{4}{|c|}{ Tiempo de Almacenamiento (Días) } \\
\hline & 1 & 3 & 6 & 9 \\
\hline Control & $65.20 \pm 7.25^{\text {b } ~}$ & $61.16 \pm 8.83$ bc & $74.55 \pm 6.93^{\text {bА }}$ & $74.64 \pm 7.30^{\mathrm{bA}}$ \\
\hline Eritorbato & $63.11 \pm 6.93^{\text {ав }}$ & $58.33 \pm 6.52^{\mathrm{ac}}$ & $70.83 \pm 6.91^{\mathrm{aA}}$ & $71.68 \pm 6.05^{\mathrm{aA}}$ \\
\hline \multicolumn{5}{|c|}{ Componente Roja $\left(a^{*}\right)$} \\
\hline Control & $-2.53 \pm 0.25$ a A & $-2.36 \pm 0.36$ а в & $-2.24 \pm 0.11$ а $\mathrm{AB}$ & $-2.98 \pm 0.89$ а Ав \\
\hline \multicolumn{5}{|c|}{ Componente Amarilla $\left(\mathrm{b}^{*}\right)$} \\
\hline Control & $20.24 \pm 2.09^{a A}$ & $20.19 \pm 2.54^{\mathrm{aA}}$ & $22.02 \pm 2.04 \mathrm{aA}$ & $22.67 \pm 1.76^{\mathrm{aA}}$ \\
\hline Ácido Cítrico & $22.58 \pm 2.59^{\mathrm{aA}}$ & $19.43 \pm 2.09^{\mathrm{aA}}$ & $24.08 \pm 2.29 \mathrm{aA}$ & $22.58 \pm 2.05^{\mathrm{aA}}$ \\
\hline Eritorbato & $21.50 \pm 2.12^{\mathrm{aA}}$ & $20.59 \pm 2.00^{\mathrm{aA}}$ & $20.75 \pm 2.18^{\text {aA }}$ & $22.36 \pm 1.80^{\text {a A }}$ \\
\hline
\end{tabular}

a. b medias con la misma letra en el mismo renglón no son significativamente $(\mathrm{P}<0.05)$ diferentes por Tratamiento.

${ }^{\text {A. }}$ B medias con la misma letra en el mismo renglón no son significativamente $(\mathrm{P}<0.05)$ diferentes por Tiempo de Almacenamiento.

la oxidación de lípidos catalizada por el grupo hemo [13], limitando la posibilidad de que el hierro de este grupo actúe como agente prooxidante. Estos resultados sugieren que la adición de antioxidantes puede ayudar a evitar otro tipo de reacciones que modifiquen el color de las salchichas cuando no están empacadas al vacío, ya que otro tipo de cambios detrimentales en salchichas conteniendo grasa vegetal pueden presentarse durante el almacenamiento. KOWALSKI, PYRCZ \& PIETRO CZYK [19] y FERREIRA et al. [20] reportaron que cambios en la oxidación de lípidos (índice de peróxido y valores TBA, respectivamente) al sustituir grasa animal con aceite de cozal y girasol, respectivamente. La adición de antioxidantes comerciales mejoró la vida de anaquel de salchichas de pollo "light" bajas en grasa formuladas con aceite vegetal, sin afectar la oxidación del colesterol [21].

En la prueba de aceptación, la mayor parte de los consumidores (68\%) reconoció que consume productos cárnicos a pesar de creer conocer el contenido de grasa animal en las salchichas. Sin embargo, el 61\% mostró interés en una salchicha con grasa vegetal, aunque solo al 50\% le pareció atractiva una coloración verde del producto. Estos resultados se vieron reflejados en la prueba de aceptación, en la que las calificaciones a los atributos evaluados estuvieron por arriba de la media de aceptación (mitad de la escala, valor de 5). La textura (8.0), la jugosidad (7.6) y el sabor (7.4) obtuvieron los atributos más altos, mientras que como era de esperarse el color fue bajo (6.2).

Si bien el oscurecimiento enzimático fue inhibido en mayor grado por la adición de los antioxidantes, ácido cítrico y eritorbato de sodio, en comparación con el control, la incorporación de las pastas sólo afectó la luminosidad final de los productos cocidos. De este modo, y debido a que el eritorbato es un ingrediente común en productos curados, el uso de este aditivo incorporado a la pasta de aguacate parece ser la mejor opción en este tipo de productos. Además, la exclusión del oxígeno o aplicación de calor inhiben las reacciones de oscurecimiento enzimático [22], por lo que el tratamiento térmico aplicado y el empaque al vacío inhibirían reacciones posteriores de oscurecimiento.

\section{CONCLUSIÓN}

La sustitución de lardo por pasta de aguacate con antioxidantes no modificó las características de color de los productos finales a las presentes condiciones de experimentación. El uso de antioxidantes como el eritorbato de sodio en la elaboración de pasta de aguacate redujo su grado de oxidación durante su almacenamiento congelado, ya que al incorporar estas pastas tratadas la vida de anaquel de este tipo de salchichas de cerdo puede ser mejorada, sin detrimento en su coloración o problemas de oxidación de lípidos, además de ser un ingrediente común en productos curados.

\section{REFERENCIAS BIBLIOGRÁFICAS}

[1] MUGUERZA, E.; FISTA, G.; ANSORENA, D.; ASTIASARAN, I.; BLOUKAS, J.G. Effect of fat level and partial replacement of pork backfat with olive oil on processing and quality characteristics of fermented sausages. Meat Sci., v. 61, n. 4, p. 397-404. 2002.

[2] SEVERI, C.; DE PILLI, T.; BAIANO, A. Partial sustitution of pork backfat with extra-virgin olive oil in 'salami' products: effects on chemical, physical and sensorial quality. Meat Sci., v. 64, n. 3, p. 323-331. 2003.

[3] KAYAARDI, S.; GÖK, V. Effect of replacing beef fat with olive oil on quality characteristics of Turkish soudjouk (sucuk). Meat Sci., v. 66, n. 1, p. 249-257. 2004.

[4] AMBROSIADIS, J.; VARELTZIS, K.P; GEORGAKIS, S.A. Physical, chemical and sensory characteristics of cooked meat emulsion style products containing vegetable oils. Int. J. Food Sci. Technol., v. 31, n. 2, p. 198-194. 1996.

[5] TAN, S.S.; AMINAH, A.; MOHD SURIA AFFANDI, Y.; ATIL, O; BABJI, A.S. Chemical, physical and sensory properties of chicken frankfurters sustituted with palm fats. Int. J. Food Sci. Nutrit., v. 52, n. 1, p. 91-98. 2001.

[6] PARK, J.; RHEE, K.S.; ZIPRIN, Y.A. Low-fat frankfurters with elevated levels of water and oleic oil. J. Food Sci., v. 55, n. 3, p. 871-872, 874. 1990.

[7] Botanical. Aguacate, grasa saludable. URL: http://www. botanical-online.com/aguacate.htm, fecha de actualización: 01/09/2005, fecha de acceso: 10/09/2005. 
[8] ESKIN, N.A.M.; HENDERSON, H.M.; TOWNSEND, R.J. Biochemistry of Foods. Orlando: Academic Press, 1971.

[9] RICHARDSON, T. Enzimas. En: FENNEMA, O.R. (Ed.) Introducción a la Ciencia de los Alimentos. Barcelona: Editorial Reverté, S.A., 1985. Cap. 6, p. 380, 382.

[10] VELÁSQUEZ ÁLVAREZ, J. Aditivos en los productos comestibles: funciones, origen y efectos secundarios. Universidad Interamericana de Puerto Rico, Recinto de Ponce, Departamento de Educación y Ciencias Sociales. URL: http: //ponce.inter.edu/cai/reserva/jvelazquez/aditivos.html. Fecha de creación: 16/11/2000, fecha de acceso: 15/08/2005.

[11] PAPADAKIS, S.E.; ABUL-MALEK, S.; KAMDEM, R.E.; YAM, K.L. A versatile and inexpensive technique for measuring color of foods. Food Technol., v. 54, n. 12, p. 48-51. 2000.

[12] YAM, K.L.; PAPADAKIS, S.E. A simple digital imaging method for measuring and analyzing color of food surfaces. J. Food Eng., v. 61, p. 137-142. 2004.

[13] PEARSON, A.M.; GIlletT, T.A. Processed Meats. Gaithersburg: Aspen Publishers, 1999.

[14] MILLER, R.K. Sensory methods to evaluate muscle foods. En: KINSMAN, D.M.; KUTOLA, A.W.; BREIDENSTEIN, B.C. (Ed.) Muscle Foods: Meat, Poultry and Seafood Technology. New York: Chapman \& Hall, 1994. Cap. 12, p. 333-377.

[15] PEDRERO, D; PANGBORN, R. Evaluación Sensorial de los Alimentos. Métodos Analíticos. Ciudad de México: Editorial Alhambra Mexicana, 1996, p. 67-102.
[16] DER, G.; EVERITT, B.S. A Handbook of Statistical Analyses Using SAS. Boca Ratón: Chapman \& Hall/ CRC, 2000.

[17] CUPPETT, S.L. The use of natural antioxidants in food products of animal origin. En: DEMAN, J.M. (Ed.) Principles of Food Chemistry. New York: Chapman \& Hall, 1999. Cap. 12, p. 285-310.

[18] POKORNÝ, J.; KORCZAK, J. Preparation of natural antioxidants. En: POKORNÝ, J.; YANISHLIEVA, N.; GORDON, M. (Ed.) Antioxidants in Food. Boca Raton: CRC Press, 2001.

[19] KOWALSKI, R.; PYRCZ, J.; PIETRO CZYK, K. Partial sustitution of animal fat with plant origin oil in the production of finely comminuted sausages. Electron J. Polish Agric. Univ., v. 1, n.1, 1998.

[20] FERRERIA, M.F; SILVA, A.T.; ROBS, P.G.; SCHMELZERNAGEL, W. Avaliação físico-química de salcicha tipo Viena com substituição de gordura animal por óleo de girassol. Braz. J. Food Technol., v. 6, n. 1, p. 1-7. 2003.

[21] MADRUGA, M.S.; FIGUEIREDO, M.J.; NUNES, M.L.; LIMA, F.M.S. Teores de cholesterol de lingüicas de franco "light" e tradicionais submetidas a differentes condições de estocagem. Ciênc. Tecnol. Aliment., v. 24. n. 4, p. 527-531. 2004.

[22] HAARD, N.F. Características de los tejidos de plantas comestibles. En: FENNEMA, O.R. (Ed.) Introducción a la Ciencia de los Alimentos. Barcelona: Editorial Reverté, S.A., 1985. Cap. 16, p. 861-867. 